1 Bollinger A, Butti P, Barras J-P, Trachsler H, Siegenthaler W. Red blood cell velocity in nailfold capillaries of man measured by a television microscopy technique. Microvasc Res 1974;7:61-72.

5 Preston FE, Sokol RJ, Lilleyman JS, Winfield DA, Blackburn EK. Cellular hyperviscosity as a cause of neurological symptoms in leukaemia. Br Med F 1978;i:476-8.

(Accepted 1 November 1982)

University Department of Medicine, Leeds General Infirmary, Leeds LS1 3EX

J E TOOKE, DM, MRCP, lecturer in medicine

D W MILLIGAN, BSC, MRCP, senior registrar in haematology

\section{Severe myocardial ischaemia induced by intravenous adrenaline}

The dose of adrenaline usually recommended for the treatment of anaphylactic shock is $0.3 \mathrm{mg}$ administered subcutaneously: an additional $0.1 \mathrm{mg}$ may be given intravenously, but intravenous doses in excess of $0.1 \mathrm{mg}$ are normally reserved for cardiac emergencies. ${ }^{1}$ We describe a case of severe myocardial ischaemia caused by $0.3 \mathrm{mg}$ adrenaline given intravenously.

\section{Case report}

A 23 year old Asiatic woman presented to her doctor with acute pharyngitis. After an intramuscular injection of 600000 units procaine penicillin she became pale and felt dizzy. Her pulse rate was 120 beats $/ \mathrm{min}$ and blood pressure $110 / 70 \mathrm{~mm} \mathrm{Hg}$. Suspecting an anaphylactic reaction, the doctor administered betamethasone $4 \mathrm{mg}$ intravenously, promethazine $25 \mathrm{mg}$ intramuscularly, and adrenaline $0.3 \mathrm{mg}$ intravenously. Immediately thereafter she developed severe retrosternal chest pain associated with sweating and dizziness. Sublingual nitroglycerine afforded no relief.

She presented to the emergency unit some five hours later, still suffering from chest pain; pulse rate was 105 beats/min and blood pressure 140/80 $\mathrm{mm} \mathrm{Hg}$. Jugular venous pressure was not raised, and her chest was clear. The apical impulse was normal in position and contour, and normal heart sounds with a grade $3 / 6$ late systolic murmur maximal at the fourth left intercostal space were heard at auscultation. Electrocardiography (figure a) showed sinus tachycardia, a normal PR interval and axis, and extensive $1.5 \mathrm{~mm}$ planar ST-segment depression while the QT interval corrected for rate was prolonged $(0.62 \mathrm{~s})$ : this reaction has been reported after administration of adrenaline. ${ }^{2}$ Total plasma calcium and protein concentrations measured at the same time were within the normal range.

She was admitted to the coronary care unit, and the chest pain continued to be severe despite treatment with sublingual nitroglycerine $(1.5 \mathrm{mg})$ and oral nifedipine $(60 \mathrm{mg})$. The pain was relieved by an intravenous infusion of nitroglycerine $(50 \mu \mathrm{g} / \mathrm{min})$, with a simultaneous decrease in the degree of ST-segment depression. Subsequent electrocardiograms showed progressive normalisation of the ST segments and the development of tall, peaked $\mathrm{T}$ waves in the chest leads (figure b).

Her condition remained stable after her pain had been relieved, and the nitroglycerine infusion was subsequently stopped. Serial estimations of the creatinine phosphokinase $\mathrm{MB}$ fraction were all within the normal range.

Results of a submaximal effort stress test incorporating a multigated equilibrium blood pool scan were normal. $M$ mode and two dimensiona
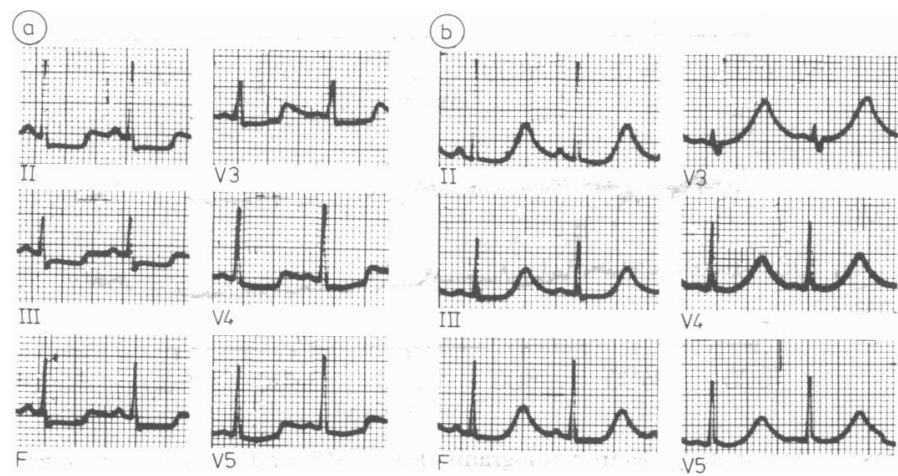

Electrocardiogram at time of admission (a) and after administration of intravenous nitroglycerine (b). echocardiography disclosed normal left ventricular wall motion and size with evidence of mild prolapse of the mitral valve. No risk factors for ischaemic heart disease could be found. She remained asymptomatic with a normal electrocardiogram six months later.

\section{Comment}

Typical ischaemic chest pain with appropriate electrocardiographic changes in a young woman with no risk factors for ischaemic heart disease after intravenous injection of adrenaline indicates that the adrenaline was almost certainly the cause of the subsequent myocardial ischaemia. This supposition was further substantiated by the results of subsequent investigations, which largely excluded the presence of preexisting ischaemic heart disease. The mechanism of myocardial ischaemia lasting for more than five hours after a single bolus of adrenaline is unclear.

The ability of catecholamines to cause myocardial damage is well established, and these hormones have also been implicated in the myocardial necrosis occurring in patients with phaeochromocytoma. ${ }^{3}$ The mechanism is thought to be either a direct effect on the myocardial cell or myocardial damage resulting from ischaemia caused by, among other things, constriction of the coronary artery. An additional mechanism whereby adrenaline may cause myocardial ischaemia is localised coronary artery spasm, as suggested by evidence showing that adrenaline added to strips of large coronary arteries increases the tension through a mechanism susceptible to alpha-antagonistic agents, ${ }^{4}$ which reverse coronary artery spasm in some patients. ${ }^{5}$

This report emphasises the potential hazards of injudicious use of adrenaline and suggests that intravenous administration of adrenaline should be confined to cardiac emergencies.

'Braunwald E. Heart disease. Philadelphia: W B Saunders, 1980:622.

2 Hecht $\mathrm{HH}$, Anderson RB. The influence of dibenamine (N, N-dibenzyl$\beta$-chloroethyl-amine) on certain functions of the sympathetic nervous system in man. Am $\mathcal{F}$ Med $1947 ; 3: 3-14$.

${ }^{3}$ Van Vliet PD, Burchell HB, Titus JL. Focal myocarditis associated with pheochromocytoma. N Engl f Med 1966;274:1102-8.

4 Toda N. Response of isolated monkey coronary arteries to catecholamines and to transmural electrical stimulation. Circ Res $1981 ; 49: 1228-36$.

5 Ricci DR, Orlick AE, Cipriano PR, Guthauer DF, Harrison DC. Altered adrenergic activity in coronary arterial spasm: insight into mechanism based on study of coronary hemodynamics and the electrocardiogram. Am ₹ Cardiol 1979;43:1073-9.

(Accepted 2 November 1982)

Cardiac Clinic, Groote Schuur Hospital, and Department of Medicine, University of Cape Town, South Africa

ADRIAN HORAK, MB, FCP(SA), registrar

RICHARD RAINE, $M B, C H B$, senior house office

LIONEL H OPIE, MD, FRCP, professor of medicine

ELWYN A LLOYD, MD, FCP(SA), senior consultant cardiologist (now at

Krannert Institute of Cardiology, Indianapolis, Indiana 46202, USA)

\section{Late development of incisional hernia: an unrecognised problem}

A long term follow up study was carried out to determine the incidence of incisional hernia after major abdominal operations; we report the results.

\section{Patients, methods, and results}

A total of 831 patients undergoing major abdominal operations at four centres in south Wales in 1972-3 were entered into a long term follow up study. Patients were examined by a single observer at one, three, and five years for the development of incisional hernia. The details and composition of the original cohort have been described elsewhere. ${ }^{1}$ Altogether 564 surviving patients were willing to enter the study at one year. Loss of patients to follow up after one year was negligible and due mainly to death and to a few patients moving out of Wales. Information was obtained from the case notes to compare the prevalence of possible aetiological factors in patients in whom hernias developed early and those in whom they developed late. The table shows the total number of new hernias.

Full details of nine possible aetiological factors were available for 38 of the patients (21 with a hernia at one year, 14 with a hernia at three years, and three with a hernia at five years). Postoperative wound infection was the only 
Development of incisional hernia

\begin{tabular}{lccc}
\hline & Year 1 & Year 3 & Year 5 \\
\hline No of patients followed up & 564 & 482 & 431 \\
No of new hernias & 28 & 14 & 6
\end{tabular}

factor for which there was a significant difference between patients who had developed a hernia by one year ( $75 \%$ wound infection) and those who developed one subsequently $(38 \%$ wound infection $)(p<0.05)$.

Factors that showed no significant difference between the two groups were sex, obesity, malnutrition, malignant disease, coexisting medical conditions, type of incision, postoperative chest infection, and suture materials used. Preliminary data from the follow up after seven years showed that a new hernia had developed in a further four patients out of the 376 patients studied.

\section{Comment}

We are unaware of any previous long term follow up of a cohort of patients undergoing abdominal surgery to determine the incidence of incisional hernia. Previous studies have usually reported the incidence of hernia at periods up to one year postoperatively, and this has been regarded as the definitive incidence. Clearly this is a serious underestimate, since the incidence may be expected to double after one year.

The use of a single observer minimised the possibility of observer error as a cause of these new findings. Three possible explanations suggest themselves. A hernia too small to be detected clinically may have been present at the time of first examination and subsequently enlarged to become obvious. Subclinical hernias may certainly be present in obese patients, but if this is the explanation it would still represent a new finding in relation to previous, shorter term studies. Secondly, mature fibrous tissue may slowly stretch and weaken with time. A third possibility is that scar tissue may be a more dynamic tissue than has previously been recognised, so that metabolic stresses on the patient might result in a catabolic effect on the dynamic equilibrium of continuing resorption and laying down of new collagen. This latter suggestion is purely speculative, since we do not have any evidence to support or refute it. Whatever the explanation, it seems that wound infection is less important in the hernias that develop late; wound infection has been well recognised in the past as the most important factor in hernias of early onset.

Studies seeking to ascertain the incidence of incisional hernia should continue for at least five years; even then, however, the figure will be an approximation because hernias may still develop in a very few cases.

Kline A, Hughes LE, Campbell H, Williams A, Zlosnick J, Leach KG Dextran 70 in the prophylaxis of thromboembolic disease after surgery: a clinically orientated randomized double-blind trial. $\mathrm{Br}$ Med $\mathcal{f} 1975$; ii : $109-12$.

(Accepted 16 November 1982)

University Department of Surgery, Welsh National School of Medicine, Cardiff CF4 4XN

K G HARDING, $\mathrm{MB}$, CHB, research fellow

$M$ MUDGE, MB, CHB, research assistant

$S$ J LEINSTER, FRCSED, lecturer (present appointment: senior lecturer in surgery, University of Liverpool)

L E HUGHES, DS, FRCS, professor

\section{Calcium antagonist withdrawal syndrome: objective demonstration with frequency-modulated ambulatory ST-segment monitoring}

Calcium ion antagonists have excited considerable interest in the treatment of all forms of angina pectoris. ${ }^{1-3}$ Once these drugs become widely available situations may arise where patients stop taking medication intentionally or otherwise, and it is important that the effects of abrupt withdrawal and its safety are clearly established. We have documented the effects of withdrawal of calcium ion antagonists by ambulatory monitoring of the ST segment with a frequencymodulated tape recorder ${ }^{4}$ and noted a clear deterioration in the signs and symptoms of myocardial ischaemia in five patients. These cases are reported to document this phenomenon, which has great practical importance.

\section{Case reports}

Case 1-A 47 year old woman presented with a history of repeated episodes of precordial pain occurring at rest and often associated with syncope. Twenty-four-hour ambulatory monitoring showed elevation of the ST segment and ventricular arrhythmias associated with symptoms. Coronary arteriography showed normal coronary arteries with spontaneous spasm of the left anterior descending artery. She was prescribed verapamil $80 \mathrm{mg}$ six-hourly and became asymptomatic. During a routine follow-up ambulatory monitoring session she overslept and omitted to take her morning dose of verapamil. She was awakened by angina, which was controlled by verapamil. The ambulatory tape showed a pronounced alteration in the electrocardiogram with tall peaked $T$ waves, peaked $P$ waves, and ST-segment elevation before, during, and after the episode of pain. She continued to be asymptomatic after this episode taking verapamil.

Case 2-A 71 year old man had chronic stable angina of three years' duration associated with triple-vessel obstructive coronary artery disease. Verapamil $120 \mathrm{mg}$ thrice daily improved his exercise tolerance to 10 minutes and reduced the frequency of his anginal episodes. Repeated clinical and exercise evaluation confirmed the stability of his symptoms and exercise tolerance. After six months of treatment his supply of verapamil tablets became exhausted and he thought that he could manage without them. After 24 hours he began to have severe angina at rest which did not respond satisfactorily to sublingual nitrates. Ambulatory tape recording showed multiple episodes of deep ST-segment depression, some of which were associated with anginal attacks. There was no change in the heart rate during these periods of pain and ST depression. Verapamil was restarted and he became asymptomatic within 48 hours.

Case 3-A 52 year old man had chronic stable angina of three years duration due to confirmed obstructive coronary artery disease. A 24-hou ambulatory ST-segment tape during placebo showed 33 episodes of pronounced ST depression with a maximum depth of $4.2 \mathrm{~mm}$; he had seven episodes of angina during this period. Diltiazem was prescribed and produced considerable improvement. After six months, the drug was withdrawn over a period of four days as a part of a controlled trial, and he was re-evaluated after stopping all treatment. He complained of recurrence of angina, and a 24-hour ambulatory ST-segment trend chart showed seven episodes of ST elevation in addition to numerous bouts of ST depression; the frequency of angina increased to nine attacks in 24 hours (figure). Diltiazem was restarted and the ST-segment elevation abolished.

Similar findings were documented in two further patients when diltiazem was withdrawn in the same controlled trial.
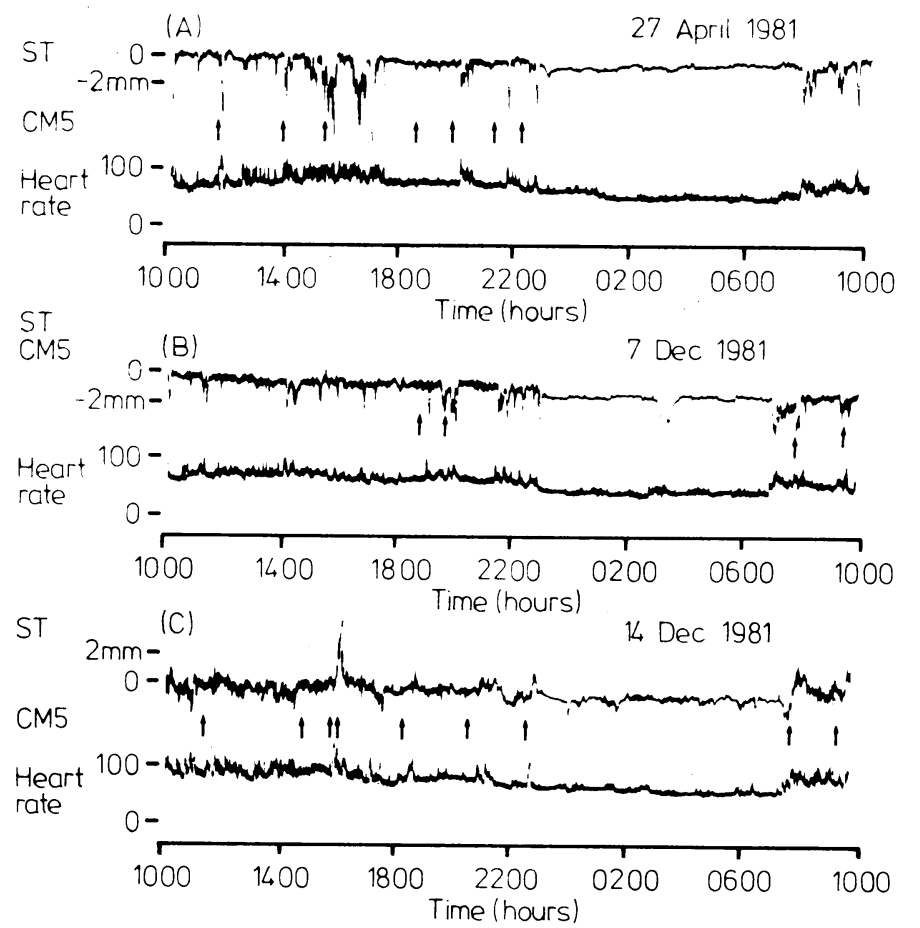

Case 3. Ambulatory electrocardiograms (lead CM5) and heart rate tracings during treatment with placebo (A) and diltiazem $120 \mathrm{mg}$ thrice daily for 24 weeks (B) and after withdrawal of diltiazem (C). Episodes of angina indicated by arrows. Calibration marker shows $2 \mathrm{~mm}$ changes. 